\title{
Niveis de Beta-Endorfina em Resposta ao Exercício e no Sobretreinamento
}

\begin{abstract}
RESUMO
O sobretreinamento (ST) é um fenômeno esportivo complexo e multifatorial; e atualmente não existe nenhum marcador independente que possa diagnosticá-lo. Interessantemente, alguns sintomas do ST apresentam relação com os efeitos da $\beta$-endorfina ( $\beta$-end ${ }^{1-31}$ ). Alguns de seus efeitos são importantes para o treinamento, como analgesia, maior tolerância ao lactato e euforia do exercício. Esses efeitos podem ser revertidos por destreinamento ou por ST, ocasionando diminuição no desempenho, redução da tolerância à carga e depressão. O exercício físico é o principal estímulo da $\beta$-end ${ }^{1-31}$, pois sua secreção é volume/intensidade dependente, tanto para exercícios aeróbios quanto anaeróbios. No entanto, o treinamento excessivo pode diminuir suas concentrações, alterando assim seus efeitos benéficos para o treinamento. Portanto, a $\beta$-end ${ }^{1-31}$ poderia ser utilizada como um marcador adicional de ST, principalmente porque seus efeitos apresentam extensa relação com os sintomas do ST. (Arq Bras Endocrinol Metab. 2008; 52/4:589-598)
\end{abstract}

Descritores: $\beta$-endorfina; Exercício; Treinamento; Sobretreinamento.

\section{ABSTRACT}

Levels of Beta-Endorphin In Response to Exercise and Overtraining.

Overtraining (OT) is a complex and multifactorial sport phenomenon, and there is no independent marker that can diagnose OT. Interestingly, some symptoms of OT are related to $\beta$-endorphin ( $\beta$-end ${ }^{1-31}$ ) effects. Some of its effects, such as analgesia, increasing lactate tolerance, and exercise-induced euphoria, are important for training. These effects can be reverted by detraining or OT, which may cause decrease in performance, reduced load tolerance, and depression. The main stimulus for $\beta$-end ${ }^{1-31}$ secretion is to exercise because its secretion is volume/intensity dependent for both aerobic and anaerobic exercise. Excesstraining, however, may reduce $\beta$-end ${ }^{1-31}$ concentrations, thus altering its beneficial effects. Therefore, $\beta$-end ${ }^{1-31}$ could be used as an additional OT marker, mainly because its effects are strongly related to OT symptoms. (Arq Bras Endocrinol Metab. 2008; 52/4:589-598)

Keywords: $\beta$-endorphin; Exercise; Training; Overtraining.

\section{INTRODUÇÃO}

Q UANDO UM TREINAMENTO EXCESSIVO e prolongado é aplicado simultaneamente à recuperação inadequada, muitas das alterações fisiológicas positivas associadas com o treinamento físico são revertidas ao sobretreinamento (ST) (1). O ST pode ser definido como o aumento no volume ou na intensidade do treinamento que resulta longo período de diminuição no desempenho (2). Visto que o ST é um desequilíbrio entre o treinamento e a

\section{revisão}

\section{GIovanI S. CUNHA}

JERRI L. RIBEIRO

Alvaro R. Oliveira

Laboratório de Pesquisa do Exercício (Lapex) da Escola de Educação Física (ESEF) da Universidade Federal do Rio Grande do Sul (UFRGS), Porto Alegre, RS, Brasil (GSC, JLR, ARO); Escola de Educação Física do Centro Universitário Metodista IPA da ESEF, Porto Alegre, RS, Brasil (JLR).

Recebido em 29/1 1/2007 Aceito em 28/03/2008 
recuperação, surge a hipótese de que o desequilíbrio neuroendócrino seja uma das suas principais razões. Vários estudos têm focalizado o hipotálamo (3-7), pois este atua na ativação do sistema nervoso autônomo, nas glândulas adrenais e também nas glândulas gonodais, resultando alterações nas catecolaminas, nos glicocorticóides e nos níveis de testosterona.

Existe outra hipótese, qual seja, que o ST seja desenvolvido pela resposta ao excessivo estresse musculoesquelético associado a períodos insuficientes de repouso e recuperação, induzindo a resposta inflamatória. Essa resposta pode evoluir para uma inflamação sistêmica, e esse processo envolve grandes quantidades de citocinas inflamatórias, como a IL-1 $\beta$, a IL-6 e o TNF- $\alpha$ (8).

Na mesma linha, Tiidus (9) aponta os radicais livres de oxigênio (RLO) como um dos fatores primários para o desenvolvimento desta resposta inflamatória induzida por lesões musculares pós-exercício e sua subseqüente recuperação. Com a presença dos RLO, os neutrófilos e os macrófagos infiltram-se no tecido muscular para recuperá-lo, gerando mais RLO (via respiratory burst), bem como citocinas que promovem a resposta inflamatória pós-exercício e a remoção do tecido lesado e sua subseqüente recuperação - mais detalhes sobre as teorias do ST em Cunha e cols. (10).

Todavia, o ST é um fenômeno complexo e multifatorial, o que dificulta a identificação de suas causas, seus sintomas e possíveis marcadores que possam identificálo. Seus sintomas confundem-se com os do pré-sobretreinamento (PST) e os do treinamento normal, sendo muitas vezes de difícil dissociação (10). Atualmente não existe nenhum marcador independente que possa prevenir ou diagnosticar o ST (1,10-13). Entretanto, o monitoramento regular do desempenho específico (atenção para a presença de fadiga crônica) e do equilíbrio entre os metabolismos anabólico e catabólico podem fornecer informações relevantes para a prevenção e a detecção do ST (10).

Algumas alterações fisio, imuno e bioquímicas, que são comumente associadas com o treinamento intenso, têm sido propostas como potenciais marcadores para a prevenção do ST. Entre elas, pode-se citar o cortisol e o lactato em resposta ao exercício intenso (14); citocinas plasmáticas como a IL-6 (15); glutamina plasmática; expressão celular $\mathrm{CD} 4+\mathrm{CD} 45 \mathrm{RO}$ e taxa CD4+/CD8; taxa neutrófilos/linfócitos; imunoglobina salivar (11); taxa testosterona/cortisol (4,6,16-20); e mais recentemente o DNA plasmático livre (21) - mais detalhes sobre os marcadores de ST em Cunha e cols. (10).

Interessantemente, alguns sinais e sintomas de ST apresentam extensa relação com os efeitos da $\beta$-endorfina $\left(\beta\right.$-end $\left.{ }^{1-31}\right)$, um hormônio peptídeo opióide endógeno secretado pela glândula hipófise anterior. Alguns de seus efeitos são muito importantes para o treinamento, como analgesia, maior tolerância ao lactato ou ao excesso de bases (22), diminuição da percepção do esforço, diminuição do desconforto muscular e respiratório (23) e, o mais importante deles, euforia do exercício (24). Esses efeitos podem ser revertidos por destreinamento ou por ST, podendo ocasionar diminuição do desempenho físico, redução da tolerância à carga e à dor, alterações no humor (25) e depressão (26).

Alguns estudos têm apresentado que o treinamento excessivo diminui as concentrações de $\beta$-end ${ }^{1-31}$ (27-30), diminuindo também seus efeitos benéficos para o treinamento físico. Dessa forma, a $\beta$-end ${ }^{1-31}$ poderia ser utilizada como marcador adicional de ST, pois sua secreção é volume/intensidade dependente, e seus efeitos apresentam extensa relação com ST. Esse hormônio possui diversos efeitos em muitas vias do metabolismo humano, alguns ainda totalmente desconhecidos. Recentemente, tem-se afirmado que a $\beta$-end ${ }^{1-31}$ pode ser secretada por células imunes, apresentando relação com as teorias mais atuais do ST (31).

Portanto, o objetivo desta revisão é descrever o comportamento da $\beta$-end ${ }^{1-31} \mathrm{em}$ resposta ao treinamento e ao exercício, seja ele máximo, aeróbio, anaeróbio ou de força, verificar as relações da $\beta$-end ${ }^{1-31}$ com o ST, bem como verificar a possibilidade de sua utilização como marcador adicional de ST.

\section{$\beta-E N D O R F I N A^{1-31}$}

A $\beta$-endorfina é um peptídeo de 31 aminoácidos $\left(\beta\right.$-end $\left.{ }^{1-31}\right)$ e foi descoberta por Li, em 1977. Tem uma potente atividade opióide e, em comparação com as encefalinas, apresenta maior resistência à degradação enzimática (32). A $\beta$-end ${ }^{1-31}$ é primariamente sintetizada na glândula hipófise anterior e clivada da pró-opiomelanocortina (POMC), seu precursor molecular, por meio das enzimas PCl e PC2 (pró-hormônio convertase) $(33,34)$.

A $\beta$-end ${ }^{1-31}$ pode ser liberada na circulação pela hipófise anterior ou projetada para áreas do cérebro por meio das fibras nervosas (22), tendo como receptores principais $\delta$ - e $\mu$-opióides receptores (35) e meia-vida plasmá- 
tica de aproximadamente 20 minutos (24). Em condições normais, apresenta concentrações muito baixas, de apenas 1-100 × 10 $0^{-12} \mathrm{M}$; embora, sob condições de estresse, como no exercício físico, aumentem 3 a 10 vezes (36).

Muitos são os efeitos atribuídos à $\beta$-end ${ }^{1-31}$, entre eles, a influência nos sistemas metabólico, imune (36), cardiovascular, respiratório, renal e reprodutivo; no ciclo menstrual (37); na amenorréia (24); na regulação da temperatura; na função gastrintestinal; no apetite; no sono; na função do aprendizado e da memória $(27,38)$; na analgesia; na diminuição do desconforto respiratório e muscular; na percepção do esforço (23); na euforia (corredor obrigatório) (24); nas alterações do humor (25); na depressão; na esquizofrenia (26); e maior tolerância ao lactato ou excesso de bases (22).

\section{RESPOSTAS DA $\beta$-END ${ }^{1-31}$ AO EXERCÍcIO}

Ainda não estão totalmente compreendidos os mecanismos que controlam a secreção da $\beta$-end ${ }^{1-31}$, mas tem sido sugerido que o estresse e o exercício físico têm um papel importante nesse processo. Tem-se sugerido que as alterações das concentrações da $\beta$-end ${ }^{1-31}$ em resposta ao exercício são volume/intensidade dependentes.

No exercício máximo e anaeróbio, extensa correlação com o lactato tem sido verificada, demonstrando aumento significativo das suas concentrações após o limiar anaeróbio ser excedido. No exercício aeróbio realizado em estado de equilíbrio entre a produção e a remoção do lactato, os níveis sangüíneos de $\beta$-end ${ }^{1-31}$ não aumentam até que a duração do exercício exceda aproximadamente uma hora, após a qual ocorre aumento exponencial (32). O balanço ácido-base ( $\mathrm{pH}$ ou níveis de ácido láctico) tem sido sugerido como mecanismo para o incremento da $\beta$-end ${ }^{1-31}$ durante o exercício.

Nesse sentido, Taylor e cols. (39) examinaram os efeitos da acidose sobre a liberação da $\beta$-end ${ }^{1-31}$ durante o exercício. Sete homens ingeriram bebida placebo ou bebida contendo 0,3 g. $\mathrm{kg}^{-1}$ de bicarbonato de sódio (processo de tamponamento) e, logo após, realizaram 20 minutos de exercício na intensidade de $85 \% \mathrm{VO}_{2 \text { máx }}$. $\mathrm{O}$ tamponamento aumentou significativamente o $\mathrm{pH}$ sangüíneo durante o repouso e durante todo o exercício quando comparado com o placebo. Os níveis de lactato foram significativamente maiores no processo de tamponamento do que no placebo. A $\beta$-end ${ }^{1-31}$ aumentou com o exercício ao longo do tempo em ambos os grupos. O excesso de base era mantido em alto nível no grupo tamponado. Os autores concluíram que a es- timulação da $\beta$-end ${ }^{1-31}$ durante o exercício devia-se à acidose metabólica, e o excesso de base o melhor indicador da liberação da $\beta$-end ${ }^{1-31}$.

Outro fator que pode estimular a $\beta$-end ${ }^{1-31}$ durante o exercício é o metabolismo ou a glicorregulação. Muitos estudos tentam elucidar o papel da $\beta$-end ${ }^{1-31}$ na homeostase da glicose durante o exercício usando opióide antagonista ou infusão direta de $\beta$-end ${ }^{1-31}$ (40-43).

Interessantemente, diversos estudos têm demonstrado que os níveis de $\beta$-end ${ }^{1-31}$ aumentam durante o exercício, seja ele máximo $(24,28,29,44-49)$, aeróbio $(24,27,46,50-55)$ ou anaeróbio $(29,39,45,47,56,57)$. No exercício de força, existe controvérsias: alguns estudos indicam aumentos significativos da $\beta$-end ${ }^{1-31}$ (58-60), outros não apresentam alterações $(61,62)$ ou até mesmo apresentam diminuição significativa (63).

Portanto, o exercício claramente induz ao aumento significativo nos níveis plasmáticos de $\beta$-end ${ }^{1-31}$. Os estudos anteriormente citados serão discutidos com mais detalhes em suas respectivas seções.

\section{RESPOSTAS DA $\beta$-END ${ }^{1-31}$ INDUZIDAS POR EXERCÍ́cIO MÁXIMO}

Os níveis de $\beta$-end ${ }^{1-31}$ são incrementados após teste máximo, em relação aos valores de repouso. A magnitude desse incremento pode alcançar de $1,5(24,28,44)$ a 7 vezes $(29,45)$ o nível de repouso.

Goldfarb e cols. (44) realizaram testes máximos com nove homens em cicloergômetro e observaram que os valores da $\beta$-end ${ }^{1-31}$ após os testes eram 1,5 vezes maiores do que os valores de repouso. Donevan e Andrew (46), após realizarem testes máximos em 19 homens, observaram aumentos de três a cinco vezes os valores de repouso. Já Rahkila e cols. (45) executaram testes máximos em esteira com cinco homens e cinco mulheres, e verificaram aumentos nas concentrações de $\beta$-end ${ }^{1-31}$ de cinco a sete vezes os valores de repouso. Resultados semelhantes foram encontrados por Kraemer e cols. (29). Neste estudo, 25 homens realizaram testes máximos em esteira e apresentaram aumentos de aproximadamente sete vezes os valores de repouso nos níveis de $\beta$-end ${ }^{1-31}(5 \mathrm{pmol} / 1$ em repouso para 37 $\mathrm{pmol} / 1$ pós-teste).

A variação desses resultados pode ser em razão de diferentes metodologias, intensidade e duração do exercício e de variação do aumento da carga (47). Alguns estudos sugerem que o exercício induz ao aumen- 
to nos níveis de $\beta$-end ${ }^{1-31}$, e esse aumento é dependente da intensidade do exercício $(45,46)$. De Meirleir e cols. (48) incluíram o lactato como parâmetro metabólico de intensidade relativa, assumindo que os níveis de $\beta$-end ${ }^{1-31}$ sejam primariamente dependentes da demanda do metabolismo anaeróbio. Foi verificado que os níveis de $\beta$-end ${ }^{1-31}$ aumentaram paralelamente aos valores de lactato até a exaustão.

Em outro estudo, 12 homens realizaram testes máximos em cicloergômetro e apresentaram aumentos de 3 vezes os valores de repouso nas concentrações de $\beta$-end ${ }^{1-31}$. Neste estudo existia a hipótese de que os níveis de $\beta$-end ${ }^{1-31}$ não aumentavam até que o limiar anae-róbio fosse excedido; após isso, aumento proporcional às concentrações de lactato era observado. Adicionalmente, a máxima concentração de $\beta$-end ${ }^{1-31}$ era correlacionada com a máxima concentração de lactato, sugerindo extensa conexão $\beta$-end ${ }^{1-31}$ com o metabolismo anaeróbio (47).

Recentemente, Heitkamp e cols. (28), após realizarem testes máximos em esteira com 23 mulheres maratonistas, verificaram aumento significativo nos níveis de $\beta$-end ${ }^{1-31}$ : os valores de repouso eram de $5 \mathrm{mmol} / \mathrm{l} \mathrm{e}$, após o teste, aumentaram para $12 \mathrm{mmol} / \mathrm{l}$. Resultados semelhantes foram observados em 14 mulheres maratonistas após teste máximo (24).

\section{RESPOSTAS DA $\beta$-END ${ }^{1-31}$ INDUZIDAS PELO EXERCÍCIO AERÓBIO}

No início dos anos 1980, diversas pesquisas sobre a intensidade e a duração do exercício e suas influências sobre os níveis plasmáticos de $\beta$-end ${ }^{1-31}$ foram realizadas. Havia controvérsia em relação à intensidade e à duração do exercício serem ou não os fatores desencadeadores da liberação da $\beta$-end ${ }^{1-31}$ no sangue periférico. Assim, vários estudos variaram a intensidade, a duração e o tipo de exercício para tentar compreender as respostas desse hormônio ao exercício.

Langefeld e cols. (64) exercitaram 10 homens a $60 \% \mathrm{VO}_{2 \text { máx }}$ por uma hora na esteira e depois na bicicleta, e observaram que não ocorreram alterações significativas nos níveis de $\beta$-end ${ }^{1-31}$. Rahkila e cols. (45) exercitaram cinco homens e cinco mulheres a $50 \%$ e $60 \% \mathrm{VO}_{2 \text { máx }}$ por 30 minutos e tampouco observaram alterações significativas. Resultados semelhantes foram encontrados por outro estudo (46), em que 19 homens pedalaram a $25 \%$ e $50 \% \mathrm{VO}_{2 \text { máx }}$, e os níveis de $\beta$-end ${ }^{1-31}$ não se alteraram; mas a $75 \% \mathrm{VO}_{2 \text { máx }}$ ocorreram aumentos significativos.
Já Goldfarb e cols. (51), exercitando indivíduos treinados nas intensidades de $60 \%, 70 \%$ e $80 \% \mathrm{VO}_{2 \operatorname{máx}}$ por 30 minutos na bicicleta, observaram aumentos significativos apenas a $70 \%$ e $80 \% \mathrm{VO}_{2 \text { máx }}$, correspondendo a 2,5 e 4,5 vezes os valores de repouso, respectivamente. Esses mesmos autores (50), utilizando anteriormente o mesmo protocolo com 12 homens não-treinados, observaram que, pedalando a $60 \% \mathrm{VO}_{2 \operatorname{máx}}$, não ocorreriam alterações significativas. Entretanto, nas intensidades de $70 \%$ e $80 \%$ $\mathrm{VO}_{2 \text { máx }}$, aumentos significativos de 2 e 5 vezes os valores pré-exercício, respectivamente, foram observados.

Outro estudo comparou 12 homens e 12 mulheres em cicloergômetro, nas intensidades de 60\% e 80\% $\mathrm{VO}_{2 \text { máx }}$, durante 25 minutos. Os resultados demonstraram que os valores entre os gêneros a $60 \%$ e $80 \%$ $\mathrm{VO}_{2 \max }$ eram semelhantes. A $60 \%$ não ocorria aumento significativo para ambos os sexos; mas a $80 \% \mathrm{VO}_{2 \max }$ ocorria aumento significativo nos níveis de $\beta$-end ${ }^{1-31}$. Os autores concluíram que não há diferenças entre homens e mulheres nessas intensidades de exercício aeróbio (52). Meyer e cols. (53) tampouco encontraram aumentos significativos em 11 mulheres que correram em intensidade de $65 \%$ a $70 \% \mathrm{VO}_{2 \operatorname{máx}}$. Em outro estudo, 10 homens realizaram exercício em cicloergômetro na intensidade de $63 \% \mathrm{VO}_{2 \text { máx }}$ por 89 minutos, e os resultados demonstraram aumento significativo apenas após 50 minutos de exercício, quando, então, ocorreu aumento exponencial (65).

$\mathrm{Na}$ base de todos esses estudos, as concentrações de $\beta$-end ${ }^{1-31}$ somente aumentavam quando a carga de trabalho era superior a $70 \% \mathrm{VO}_{2 \text { máx }}$ ou, mais precisamente, quando o limiar de lactato de $4 \mathrm{mmol} / \mathrm{L}$ era excedido. Quando o exercício aeróbio era realizado no estado de equilíbrio entre a produção e a remoção do lactato, os níveis sangüíneos de $\beta$-end ${ }^{1-31}$ não aumentavam até que a duração do exercício excedesse aproximadamente uma hora, ocorrendo incremento exponencial depois disso (32). Essas descobertas causaram conflitos, porque nesses estudos a duração do exercício ficou limitada a 60 minutos, e aumentos nos níveis de $\beta$-end ${ }^{1-31}$ em exercício aeróbio extenuante não poderiam ser excluídos.

Nessa ótica, diversas pesquisas estudaram os efeitos do exercício aeróbio de longa duração, principalmente em maratonas, para tentar elucidar os efeitos desse tipo de exercício nos níveis plasmáticos de $\beta$-end ${ }^{1-31}$. Heitkamp e cols. (24) compararam 14 maratonistas em teste máximo e maratona. No teste máximo, ocorreram aumentos de 1,9 vezes os valores de repouso da $\beta$-end ${ }^{1-31}$; 
já na maratona com duração de aproximadamente 3 horas e 22 minutos, aumentos significativos da $\beta$-end $d^{1-31}$ só foram observados após 30 minutos de recuperação.

Outro estudo avaliou 14 homens numa maratona com 4 horas de duração. Terminada a maratona, observou-se aumento significativo de 2,8 vezes os valores de repouso $(14,9$ para $42,2 \mathrm{pM} / 1, \mathrm{p}<0,001)$. Os autores concluíram que o exercício submáximo de grande duração induz ao aumento significativo nos níveis plasmáticos de $\beta$-end ${ }^{1-31}$, e o volume do exercício pode ser um dos principais fatores para sua secreção (54). Tal afirmação é reforçada por outros autores $(27,55)$, que também observaram que o exercício aeróbio extenuante produz aumentos significativos nas concentrações da $\beta$-end ${ }^{1-31}$

Portanto, tanto a intensidade quanto a duração do exercício são importantes para a estimulação da secreção da $\beta$-end ${ }^{1-31}$, ficando evidente que os exercícios de alta intensidade necessitam de menor duração comparados a exercícios de baixa intensidade (menor que $60 \% \mathrm{VO}_{2 \text { máx }}$ ), os quais necessitam de duração superior a 1 hora.

\section{RESPOSTAS DA $\beta$-END ${ }^{1-31}$ INDUZIDAS POR EXERCÍCIO ANAERÓBIO}

O exercício anaeróbio de alta intensidade e com 30 segundos de duração estimula a liberação de $\beta$-end ${ }^{1-31}$ por vários minutos. $\mathrm{O}$ pico máximo nos níveis sangüíneos periféricos é de aproximadamente duas a quatro vezes maior do que em repouso (47). Dois estudos com protocolos de exercícios semelhantes na esteira (45) e no cicloergômetro (47), com duração de um minuto e com concentração máxima de lactato entre 12 e 15 $\mathrm{mmol} / \mathrm{l}$, resultaram aumentos idênticos nos níveis plasmáticos de $\beta$-end ${ }^{1-31}$, alcançando duas vezes os valores de repouso. Nos estudos em que a duração do exercício era maior, resultava aumentos dos níveis de $\beta$-end ${ }^{1-31}$ mais pronunciados $(29,56)$.

Taylor e cols. (39) estudaram os efeitos do metabolismo anaeróbio sobre as respostas da $\beta$-end ${ }^{1-31}$. Os sujeitos corriam na esteira a $85 \% \mathrm{VO}_{2 \text { máx }}$, e verificou-se incremento significativo nos níveis de $\beta$-end ${ }^{1-31}$ de 40 $\mathrm{pg} / \mathrm{ml}$ em repouso para $110 \mathrm{pg} / \mathrm{ml}$ após o teste.

A conexão entre o aumento dos níveis de $\beta$-end ${ }^{1-31}$ e o metabolismo anaeróbio é também indicada por positiva correlação entre concentrações máximas de $\beta$-end ${ }^{1-31}$ e lactato (47), na qual se hipotetiza que alguma substância do metabolismo anaeróbio possa estimular o eixo hipotálamo-hipófise.

\section{RESPOSTAS DA $\beta$-END ${ }^{1-31}$ INDUZIDAS PELO EXERCÍCIO DE FORÇA}

Existe pouca informação sobre as respostas da $\beta$-end ${ }^{1-31}$ ao exercício de força, e essas respostas são contraditórias. Pierce e cols. (61) reportaram que não havia alterações significativas nos níveis de $\beta$-end ${ }^{1-31} \mathrm{em}$ seis homens treinados, após a execução de três séries de oito repetições, a $80 \%$ da repetição máxima (1 RM). Em contraste, esses mesmos autores depois verificaram que os níveis de $\beta$-end ${ }^{1-31}$ diminuíam significativamente após a realização de um protocolo similar de exercício de força aplicado em estudantes (63). Outro estudo, em que sete homens treinados executaram um protocolo de três séries de 10 RM com quatro exercícios diferentes, demonstrou que um baixo volume de exercício de força não aumenta significativamente os níveis de $\beta$-end ${ }^{1-31}(62)$.

Por outro lado, Kraemer e cols. (58) demonstraram que oito homens treinados somente aumentavam suas concentrações de $\beta$-end ${ }^{1-31}$ com a execução de um protocolo com elevada carga de trabalho (duas séries de 10 RM com 1 minuto de repouso entre as séries). Foi verificado que as respostas da $\beta$-end ${ }^{1-31}$ são diferentes para cada tipo de protocolo, e que não há diferenças significativas entre os valores do pré-exercício e os valores de 24 e 48 horas pós-exercício. Os autores sugerem que a duração da série, o intervalo de repouso e a produção total de força influenciam as respostas da $\beta$-end ${ }^{1-31}$. Esse mesmo grupo de autores, previamente já havia demonstrado que a $\beta$-end ${ }^{1-31}$ era significativamente aumentada em 28 levantadores de peso, após a realização de trabalho de intensidade moderada-alta (59).

\section{RESPOSTAS DA $\beta$-END ${ }^{1-31}$ INDUZIDAS PELO TREINAMENTO}

Na tentativa de verificar o comportamento temporal da secreção da $\beta$-end ${ }^{1-31}$, foram comparados seis ciclistas treinados com seis não-treinados, em três exercícios com diferentes intensidades. $\mathrm{O}$ exercício aeróbio era realizado em cicloergômetro nas intensidades de $60 \%$, $70 \%$ e $80 \% \mathrm{VO}_{2 \text { máx }}$, por 30 minutos para cada intensidade. Os resultados demonstraram que a $60 \%$ não ocorreram mudanças significativas; e a $70 \%$ e $80 \%$ ocorreram incrementos de 2,5 e 4,5 vezes os valores de repouso, respectivamente. $\mathrm{O}$ nível de treinamento não influenciou na secreção da $\beta$-end ${ }^{1-31}$, e o comportamento temporal foi semelhante para ciclistas treinados e não treinados. Tampouco ocorreram diferenças significati- 
vas nos valores de repouso da $\beta$-end ${ }^{1-31}$ entre treinados $(4,61 \mathrm{pmol} / \mathrm{l})$ e não-treinados $(4,03 \mathrm{pmol} / \mathrm{l})(5 \mathrm{l})$.

Fourier e cols. (55) avaliaram as alterações nos níveis de $\beta$-end ${ }^{1-31}$ em 11 maratonistas treinados e sete homens não-treinados durante uma ultramaratona (distância de 110 km e duração de 10 a 20 horas), em que foi verificado que o grupo de maratonistas treinados apresentou aumento significativamente maior do que o do grupo não-treinado $(\mathrm{p}=0,001)$. Também se constatou que não havia relação entre o status de treinamento em termos de $\mathrm{km} / \mathrm{semana}$ ou o tempo de realização da prova com os níveis plasmáticos de $\beta$-end ${ }^{1-31}$.

Kraemer e cols. (29) examinaram os efeitos de três diferentes programas de treinamento nos níveis plasmáticos de $\beta$-end ${ }^{1-31}$ após a realização de teste máximo em esteira. O treinamento foi dividido em três grupos, oito indivíduos realizaram treinamento de sprint (três vezes por semana, duas séries de quatro sprints máximos); 10 indivíduos realizaram treinamento de endurance (três vezes por semana, 30 minutos a $80 \% \mathrm{VO}_{2 \text { máx }}$ ); e sete indivíduos realizaram o treinamento de endurance somado ao treinamento de sprint (seis vezes por semana). Concluiu-se que no pré-treino não existiam diferenças significativas entres os grupos. O exercício máximo induziu ao aumento significativo nos níveis de $\beta$-end ${ }^{1-31}$, tanto no pré quanto no pós-treino, em todos os grupos. O grupo sprint apresentou aumento significativo após o treinamento nas concentrações de $\beta$-end ${ }^{1-31}$. O grupo de endurance não apresentou mudanças significativas nas concentrações de $\beta$-end ${ }^{1-31}$ após o treinamento. Por outro lado, o grupo que somou os treinamentos apresentou redução significativa nas concentrações de $\beta$-end ${ }^{1-31}$ após o treinamento. Esses dados sugerem que diferentes programas de treinamento de corrida provocam diferentes efeitos sobre a secreção de $\beta$-end ${ }^{1-31}$, e que o treinamento excessivo pode ocasionar saturação da secreção de $\beta$-end ${ }^{1-31}$ pelo eixo hipotálamo-hipófise. Esta hipótese foi confirmada por outro estudo, no qual indivíduos diagnosticados com ST apresentavam diminuição significativa dos níveis de $\beta$-end ${ }^{1-31}$ em comparação com o grupo-controle (66).

Em outro estudo (59), foram avaliados 28 homens treinados em levantamento de peso olímpico, na intensidade moderada-alta e com baixo volume (15 jump vertical e $3 \times 10$ snatch pulls). Foi observado que os níveis de $\beta$-end ${ }^{1-31}$ aumentaram significativamente do pré para o pós-exercício, e não foram encontradas diferenças significativas entre os estilos de levantamento. $\mathrm{O}$ tempo de treinamento e o nível de força não causaram diferenças significativas nas concentrações de $\beta$-end ${ }^{1-31}$.

Heitkamp e cols. (28) observaram as alterações da $\beta$-end ${ }^{1-31}$ em resposta ao treinamento de endurance em 23 mulheres treinadas, utilizando teste máximo na esteira. O treinamento consistia de correr 30 minutos no limiar anaeróbio, três vezes por semana, durante oito semanas. Foi observado que os níveis basais de $\beta$-end ${ }^{1-31}$ não se alteraram após o treinamento, o pico de $\beta$-end ${ }^{1-31}$ diminuiu significativamente em relação ao pré-treino, e os valores de recuperação estavam significativamente diminuídos em relação ao pré-treino. Os autores concluíram que o treinamento de endurance modula as respostas da $\beta$-end ${ }^{1-31}$.

Também foram verificados os efeitos de uma maratona (três horas de duração) em altas altitudes, em 14 maratonistas treinados. Foi observado que os níveis plasmáticos de $\beta$-end ${ }^{1-31}$ aumentaram significativamente após a maratona, e os atletas mais jovens tiveram aumento superior. Esses mesmos atletas já haviam participado da prova em duas edições anteriores; assim, longitudinalmente, as alterações da $\beta$-end ${ }^{1-31}$ nesses três anos foram: $1^{\mathrm{a}}, 300 \%, 2^{\mathrm{a}}, 140 \%$ e $3^{\mathrm{a}}, 110 \%$, ocorrendo significativa diminuição no percentual de secreção da $\beta$-end ${ }^{1-31}$. A magnitude do incremento era dependente da idade; o tempo de exposição a altas atitudes foi associado a incremento significativo nas concentrações de repouso da $\beta$-end ${ }^{1-31}$; já o exercício crônico foi associado à diminuição da secreção da $\beta$-end ${ }^{1-31}$ (27).

Lobstein e Ismael (30) compararam 30 homens em relaçãoàs suas concentrações de $\beta$-end ${ }^{1-31} / \beta$-lipotropina. Os indivíduos foram divididos em grupos: 10 indivíduos treinados por quatro meses em fitness (treinamento aeróbio); 10 indivíduos que realizavam caminhadas; e 10 sedentários. Foi observado que, em repouso, não ocorreram mudanças significativas entre os grupos no pré-teste; mas, no grupo treinado, os níveis de repouso da $\beta$-end $d^{1-31} / \beta$-lipotropina diminuíram significativamente do pré-teste para o pós-teste: de 11,02 pmol/1 para 7,22 pmol/1, respectivamente. Os autores concluíram que quatro meses de treinamento aeróbio reduzem significativamente as concentrações de repouso da $\beta$-end ${ }^{1-31} / \beta$-lipotropina.

No treinamento, as respostas da $\beta$-end ${ }^{1-31}$ variam dependendo principalmente do tipo de treinamento a ser executado: aeróbio, anaeróbio ou de força. Interessantemente, o treinamento crônico diminui as concentrações plasmáticas de $\beta$-end ${ }^{1-31}(27-30,66)$, muito 
provavelmente em virtude de saturação da secreção de $\beta$-end ${ }^{1-31}$.

\section{PRINCIPAIS MARCADORES DE SOBRETREINAMENTO: VANTAGENS E DESVANTAGENS}

Tendo em vista que, existe constantemente risco de desequilíbrio entre treinamento, competição e recuperação, qualquer atleta está sujeito ao ST. Nesse contexto, vários estudos foram realizados no intuito de identificar causas, sintomas, hipóteses e marcadores que pudessem identificá-lo. No entanto, esse diagnóstico é muito difícil, pois os sintomas do ST se confundem com os do PST e com os do treinamento normal, o que dificulta seu diagnóstico (10). O único tratamento conhecido é a diminuição no volume de treinamento ou repouso completo. O atleta que tenha desenvolvido o ST deve repousar de seis a 12 semanas $(67)$. Atualmente não existe nenhum marcador independente que possa prevenir ou diagnosticar o ST (1,10-13). A avaliação da diminuição do desempenho específico do esporte (atenção para a presença de fadiga crônica) ainda representa o padrãoouro no diagnóstico do ST e necessita de testes específicos para cada esporte (10).

Nesse sentido, testes máximos até a exaustão podem identificar diminuição no desempenho específico do esporte, pois atletas com ST normalmente apresentam diminuição no desempenho anaeróbico láctico, redução no tempo de exaustão em testes de alta intensidade e pequena diminuição na freqüência cardíaca máxima. $\mathrm{O}$ lactato também apresenta diminuição durante o exercício submáximo. Conseqüentemente, algumas dessas alterações fisiológicas, bioquímicas e imunológicas, comumente associadas ao treinamento intenso, têm sido propostas como potenciais marcadores do ST. Entre os principais marcadores, pode-se citar o cortisol e o lactato em resposta ao exercício intenso (14); citocinas plasmáticas, como a IL-6 (15); glutamina plasmática; expressão celular CD4+CD45RO e taxa CD4+/CD8; taxa neutrófilos/linfócitos; imunoglobina salivar (11); taxa testosterona/cortisol $(4,6,16-20)$ e mais recentemente o DNA plasmático livre (21) - mais detalhes sobre os marcadores de ST em Cunha e cols. (10).

No que diz respeito a desvantagens e vantagens dos principais marcadores bioquímicos, hormonais e imunológicos do ST, além da desvantagem do elevado custo financeiro, muitos destes marcadores não respondem especificamente ao ST e podem ser influencia- dos por outros fatores que não necessariamente tem relação como ST.

A concentração plasmática de glutamina pode diminuir após trauma físico, queimaduras, inflamações e infecções e aumentar temporariamente após a ingestão de alimentos protéicos (11), por outro lado, baixos níveis de glutamina plasmática são normalmente reportados em atletas com ST.

Há certa dificuldade na análise do lactato, pois tanto na adaptação ao treinamento normal quanto no ST existe deslocamento da curva do lactato para a direita, dificultando a dissociação dos efeitos do treinamento para os efeitos do ST (10). Entretanto, atletas com ST apresentam menor produção de lactato durante testes máximos (14).

A razão testosterona/cortisol ou testosterona-livre/ cortisol representa o equilíbrio entre os estados anabólico e catabólico do organismo, sendo sugerido que se a diminuição desta taxa for superior a $30 \%$ o atleta seria diagnosticado com ST (17), entretanto, deve ser levado em conta o princípio da individualidade e o desempenho específico do atleta, pois não necessariamente quando a diminuição da razão for superior a $30 \%$ o atleta terá diminuição em seu desempenho específico.

O DNA plasmático livre embora apresente consistente correlação com as cargas de treinamento durante períodos de altíssimo volume de treinamento, ainda não demonstra ser um marcador específico de ST, pois sua origem no plasma durante o exercício ainda não é conhecida (21).

\section{RELAÇÕES DA $\beta$-END ${ }^{1-31}$ COM $O$ SOBRETREINAMENTO}

Além das influências do treinamento e do exercício sobre a secreção de $\beta$-end ${ }^{1-31}$ (volume/intensidade dependentes), o ST também está associado às alterações no sistema dos opióides endógenos, mais especificamente em relação a $\beta$-end ${ }^{1-31}$. O ST apresenta redução na produção de lactato e intolerância à acidose, e provavelmente está relacionado com a limitação ou a saturação da capacidade da secreção de $\beta$-end ${ }^{1-31}$ (32).

O ST provoca desequilíbrio neuroendócrino (3-7), mais precisamente no eixo hipotálamo-hipófise. Tendo em vista que a secreção $\beta$-end ${ }^{1-31}$ é dependente desse eixo, utilizá-la como marcador de ST parece ser lógico, ainda mais que sua secreção é diminuída em resposta ao treinamento excessivo $(27-30,66)$ e seus efeitos têm extensa relação com os sinais e os sintomas do ST. 
Muito provavelmente, uma inabilidade do eixo hipotálamo-hipófise de responder adequadamente ao estresse excessivo ou ao ST, assim, provocando a inibição da secreção de $\beta$-end ${ }^{1-31}$, conforme verificado nos estudos anteriormente citados. Essa diminuição da secreção de $\beta$-end ${ }^{1-31}$ pode reverter seus efeitos benéficos, como analgesia, maior tolerância ao lactato (22), diminuição do desconforto muscular e da percepção do esforço (23) e, principalmente, euforia do exercício (24), que é extremamente necessária para a manutenção do treinamento intenso. Esses efeitos podem ser revertidos pelo treinamento extenuante ou por ST, podendo ocasionar diminuição do desempenho físico, redução da tolerância à carga e à dor muscular, alterações no humor (25) e depressão (26), sintomas evidentes durante o ST.

Curiosamente, a $\beta$-end ${ }^{1-31}$ também está relacionada com as teorias mais atuais do ST $(8,15)$, mais especificamente com a teoria do trauma musculoesquelético e sua subseqüente resposta inflamatória. Esta resposta pode ter com seus fatores primários a IL-6 (15) e principalmente os RLO (9), em que os neutrófilos e os macrófagos na presença dos RLO infiltram-se no tecido muscular para recuperá-lo, gerando mais RLO, bem como citocinas que promovem a resposta inflamatória pós-exercício e a remoção do tecido lesado e sua subseqüente recuperação $(9,68)$.

Quando a produção de RLO excede a habilidade antioxidante dos tecidos, o resultado é o estresse oxidativo, que é associado a lesões musculares, fadiga e diminuição de força $(10,68)$. Por outro lado, o exercício induz a adaptações positivas que protegem o organismo dos danos gerados pelos RLO, como demonstrado em nossos estudos $(69,70)$, apresentando maior atividade da enzima glutationa peroxidase em triatletas comparados com indivíduos não-treinados e capacidade antioxidante total plasmática (TRAP) aumentada em ambos os grupos. Entretanto, o treinamento crônico pode interferir no sistema antioxidante, gerando desequilíbrio entre a produção de RLO e a resposta antioxidante, causando, assim, estresse oxidativo crônico ou resposta inflamatória sistêmica induzida por estresse oxidativo, que pode diminuir o desempenho físico e levar ao desenvolvimento do ST (10).

Recentemente, tem-se demonstrado que a $\beta$-end ${ }^{1-31}$ pode ser secretada por células imunes (31), aumentando também a atividade das células natural killer durante situações de estresse (36), demonstrando ter papel importante no sistema inume. Entretanto, muitos estu- dos ainda são necessários para compreender totalmente os efeitos da $\beta$-end ${ }^{1-31}$, principalmente sob o foco do sistema imune.

\section{CONSIDERAÇÕES FINAIS}

As respostas da $\beta$-end ${ }^{1-31}$ ao exercício e ao treinamento estão bem demonstradas. Dessa forma, sua utilização como marcador de ST é totalmente coerente, ainda mais que seus efeitos apresentam extensa relação com os sinais e sintomas do ST.

Atualmente, ainda não existe nenhum marcador independente que possa prevenir ou diagnosticar o ST, assim, recomenda-se a utilização da $\beta$-end ${ }^{1-31}$ como marcador adicional de ST, mensurando seus níveis periodicamente ao longo das etapas do treinamento por meio de protocolos padronizados, concomitantemente com mensurações de outros marcadores bioquímicos e hormonais. Salientando que em uma situação em que ocorra diminuição do desempenho físico, paralelamente diminuição das concentrações da $\beta$-end ${ }^{1-31}$ e o surgimento dos sintomas do ST, estas evidências em conjunto sugerem fortemente a possibilidade do desenvolvimento do ST.

\section{AGRADECIMENTOS}

A Márcia Dornelles pela revisão do manuscrito, ao CNPq, Capes e Cenesp pelo financiamento para o desenvolvimento deste trabalho.

\section{REFERÊNCIAS}

1. Armstrong LE, VanHeest JL. The unknown mechanism of the overtraining syndrome: clues from depression and psychoneuroimmunology. Sports Med. 2002;32(3):185-209.

2. Fry $A C$, Kraemer WJ. Resistance exercise overtraining and overreaching. Neuroendocrine responses. Sports Med. 1997;23(2):106-29.

3. Fry AC, Kraemer WJ, Ramsey LT. Pituitary-adrenal-gonadal responses to high-intensity resistance exercise overtraining. $J$ Appl Physiol. 1998;85(6):2352-9.

4. Hug M, Mullis PE, Vogt M, Ventura N, Hoppeler H. Training modalities: over-reaching and over-training in athletes, including a study of the role of hormones. Best Pract Res Clin Endocrinol Metab. 2003;17(2):191-209.

5. Meeusen R, Piacentini MF, Busschaert B, Buyse L, De Schutter G, Stray-Gundersen J. Hormonal responses in athletes: the use of a two bout exercise protocol to detect subtle differences in (over)training status. Eur J Appl Physiol. 2004;91(2-3):140-6. 
6. Mujika I, Chatard JC, Padilla S, Guezennec CY, Geyssant A Hormonal responses to training and its tapering off in competitive swimmers: relationships with performance. Eur J Appl Physiol Occup Physiol. 1996;74(4):361-6.

7. MacKinnon LT. Special feature for the olympics: effects of exercise on the immune system: overtraining effects on immunity and performance in athletes. Immunol Cell Biol. 2000;78(5):502-9.

8. Smith LL. Cytokine hypothesis of overtraining: a physiological adaptation to excessive stress? Med Sci Sports Exerc. 2000;32(2):317-31.

9. Tiidus PM. Radical species in inflammation and overtraining. Can J Physiol Pharmacol. 1998;76(5):533-8.

10. Cunha GS, Ribeiro JL, Oliveira AR. Sobretreinamento: teorias, diagnóstico e marcadores. Rev Bras Med Esporte. 2006;12(5):297-302.

11. Gleeson M. Biochemical and immunological markers of overtraining. Journal of Sports Science and Medicine. 2002;2:31-41.

12. Petibois C, Cazorla G, Poortmans JR, Deleris G. Biochemical aspects of overtraining in endurance sports: a review. Sports Med. 2002;32(13):867-78.

13. Lac G, Maso F. Biological markers for the follow-up of athletes throughout the training season. Pathol Biol (Paris). 2004;52(1):43-9.

14. Bosquet $L$, Leger $L$, Legros $P$. Blood lactate response to overtraining in male endurance athletes. Eur J Appl Physiol. 2001;84(1-2):107-14.

15. Robson P. Elucidating the unexplained underperformance syndrome in endurance athletes: the interleukin- 6 hypothesis. Sports Med. 2003;33(10):771-81.

16. Budgett R. Fatigue and underperformance in athletes: the overtraining syndrome. Br J Sports Med. 1998;32(2):107-10.

17. Adlercreutz $H$, Harkonen M, Kuoppasalmi K, Naveri H, Huhtaniemi I, Tikkanen $\mathrm{H}$, et al. Effect of training on plasma anabolic and catabolic steroid hormones and their response during physical exercise. Int J Sports Med. 1986;7 Suppl 1:27-8.

18. Filaire E, Bernain X, Sagnol M, Lac G. Preliminary results on mood state, salivary testosterone:cortisol ratio and team performance in a professional soccer team. Eur J Appl Physiol. $2001 ; 86(2): 179-84$.

19. Gorostiaga EM, Izquierdo M, Ruesta M, Iribarren J, GonzalezBadillo JJ, lbanez J. Strength training effects on physical performance and serum hormones in young soccer players. Eur $\mathrm{J}$ Appl Physiol. 2004;91(5-6):698-707.

20. Hoffman JR, Falk B, Radom-Isaac S, Weinstein Y, Magazanik A, Wang $Y$, et al. The effect of environmental temperature on testosterone and cortisol responses to high intensity, intermittent exercise in humans. Eur J Appl Physiol Occup Physiol. 1997;75(1):83-7.

21. Fatouros IG, Destouni A, Margonis $K$, Jamurtas AZ, Vrettou $C$ Kouretas D, et al. Cell-free plasma DNA as a novel marker of aseptic inflammation severity related to exercise overtraining. Clin Chem. 2006;52(9):1820-4.

22. Goldfarb $A H$, Jamurtas $A Z$. Beta-endorphin response to exercise. An update. Sports Med. 1997;24(1):8-16.

23. Sgherza AL, Axen K, Fain R, Hoffman RS, Dunbar CC, Haas F. Effect of naloxone on perceived exertion and exercise capacity during maximal cycle ergometry. J Appl Physiol. 2002;93(6):2023-8.

24. Heitkamp HC, Huber W, Scheib K. beta-Endorphin and adrenocorticotrophin after incremental exercise and marathon running-female responses. Eur J Appl Physiol Occup Physiol. $1996 ; 72(5-6): 417-24$.
25. Farrell PA. Exercise and endorphins-male responses. Med Sci Sports Exerc. 1985;17(1):89-93.

26. De Cree C. The possible involvement of endogenous opioid peptides and catecholestrogens in provoking menstrual irregularities in women athletes. Int $\mathrm{J}$ Sports Med. 1990;11(5):329-48.

27. Appenzeller O, Wood SC. Peptides and exercise at high and low altitudes. Int J Sports Med. 1992;13 Suppl 1:S135-40.

28. Heitkamp HC, Schulz H, Rocker K, Dickhuth HH. Endurance training in females: changes in beta-endorphin and ACTH. Int J Sports Med. 1998;19(4):260-4.

29. Kraemer WJ, Fleck SJ, Callister R, Shealy M, Dudley GA, Maresh $\mathrm{CM}$, et al. Training responses of plasma beta-endorphin adrenocorticotropin, and cortisol. Med Sci Sports Exerc. $1989 ; 21(2): 146-53$.

30. Lobstein DD, Ismail AH. Decreases in resting plasma betaendorphin/-lipotropin after endurance training. Med Sci Sports Exerc. 1989;21(2):161-6.

31. Rittner HL, Stein C. Involvement of cytokines, chemokines and adhesion molecules in opioid analgesia. Eur $\mathrm{J}$ Pain. 2005;9(2):109-12.

32. Schwarz L, Kindermann W. Changes in beta-endorphin levels in response to aerobic and anaerobic exercise. Sports Med. 1992;13(1):25-36

33. Wei S, Feng Y, Kalinina E, Fricker LD. Neuropeptide-processing carboxypeptidases. Life Sci. 2003;73(6):655-62.

34. Zhou A, Webb G, Zhu X, Steiner DF. Proteolytic processing in the secretory pathway. J Biol Chem. 1999;274(30):20745-8.

35. Narita M, Tseng LF. Evidence for the existence of the beta-endorphin-sensitive "epsilon-opioid receptor" in the brain: the mechanisms of epsilon-mediated antinociception. Jpn J Pharmacol. 1998;76(3):233-53.

36. Pedersen BK, Hoffman-Goetz L. Exercise and the immune system: regulation, integration, and adaptation. Physiol Rev. 2000;80(3):1055-81.

37. McArthur JW. Endorphins and exercise in females: possible connection with reproductive dysfunction. Med Sci Sports Exerc. 1985;17(1):82-8.

38. Sforzo GA. Opioids and exercise. An update. Sports Med. 1989;7(2):109-24.

39. Taylor DV, Boyajian JG, James N, Woods D, Chicz-Demet A Wilson $A F$, et al. Acidosis stimulates beta-endorphin release during exercise. J Appl Physiol. 1994;77(4):1913-8.

40. Angelopoulos TJ, Denys BG, Weikart C, Dasilva SG, Michae TJ, Robertson RJ. Endogenous opioids may modulate catecholamine secretion during high intensity exercise. Eur J Appl Physiol Occup Physiol. 1995;70(3):195-9.

41. Angelopoulos TJ, Robertson RJ, Goss FL, Utter A. Insulin and glucagon immunoreactivity during high-intensity exercise under opiate blockade. Eur J Appl Physiol Occup Physiol. 1997;75(2):132-5.

42. Hickey MS, Trappe SW, Blostein AC, Edwards BA, Goodpaster $B$, Craig BW. Opioid antagonism alters blood glucose homeostasis during exercise in humans. J Appl Physiol. 1994;76(6):2452-60.

43. Farrell PA, Gustafson AB, Garthwaite TL, Kalkhoff RK, Cowley AW, Morgan WP. Influence of endogenous opioids on the response of selected hormones to exercise in humans. J App Physiol. 1986;61(3):1051-7.

44. Goldfarb AH, Hatfield BD, Sforzo GA, Flynn MG. Serum betaendorphin levels during a graded exercise test to exhaustion. Med Sci Sports Exerc. 1987;19(2):78-82. 
45. Rahkila P, Hakala E, Salminen K, Laatikainen T. Response of plasma endorphins to running exercises in male and female endurance athletes. Med Sci Sports Exerc. 1987;19(5):451-5.

46. Donevan $\mathrm{RH}$, Andrew GM. Plasma beta-endorphin immunoreactivity during graded cycle ergometry. Med Sci Sports Exerc. 1987;19(3):229-33.

47. Schwarz L, Kindermann W. Beta-endorphin, adrenocorticotropic hormone, cortisol and catecholamines during aerobic and anaerobic exercise. Eur J Appl Physiol Occup Physiol. 1990;61(3-4):165-71

48. De Meirleir K, Naaktgeboren N, Van Steirteghem A, Gorus F, Olbrecht J, Block P. Beta-endorphin and ACTH levels in peripheral blood during and after aerobic and anaerobic exercise. Eur J Appl Physiol Occup Physiol. 1986;55(1):5-8.

49. Bouix O, Brun JF, Fedou C, Raynaud E, Kerdelhue B, Lenoir V, et al. Plasma beta-endorphin, corticotrophin and growth hormone responses to exercise in pubertal and prepubertal children. Horm Metab Res. 1994;26(4):195-9.

50. Goldfarb AH, Hatfield BD, Armstrong D, Potts J. Plasma betaendorphin concentration: response to intensity and duration of exercise. Med Sci Sports Exerc. 1990;22(2):241-4.

51. Goldfarb AH, Hatfield BD, Potts J, Armstrong D. Beta-endorphin time course response to intensity of exercise: effect of training status. Int J Sports Med. 1991;12(3):264-8.

52. Goldfarb AH, Jamurtas AZ, Kamimori GH, Hegde S, Otterstetter R, Brown DA. Gender effect on beta-endorphin response to exercise. Med Sci Sports Exerc. 1998;30(12):1672-6.

53. Meyer WR, Muoio D, Hackney TC. Effect of sex steroids on beta-endorphin levels at rest and during submaximal treadmill exercise in anovulatory and ovulatory runners. Fertil Steril. 1999;71(6):1085-91.

54. Estorch M, Fuente T, Serra-Grima R, Flotats A, Berna L, Sanz D, et al. The effect of a race 4 hours in duration on the production of beta-endorphin and adrenocorticotropic hormone. Med Clin (Barc). 1998;111(20):770-3.

55. Fournier PE, Stalder J, Mermillod B, Chantraine A. Effects of a 110 kilometers ultra-marathon race on plasma hormone levels. Int J Sports Med. 1997;18(4):252-6.

56. Farrell PA, Kjaer M, Bach FW, Galbo H. Beta-endorphin and adrenocorticotropin response to supramaximal treadmill exercise in trained and untrained males. Acta Physiol Scand. 1987;130(4):619-25.

57. Schulz A, Harbach H, Katz N, Geiger L, Teschemacher H. betaEndorphin immunoreactive material and authentic beta-endorphin in the plasma of males undergoing anaerobic exercise on a rowing ergometer. Int J Sports Med. 2000;21(7):513-7.

58. Kraemer WJ, Dziados JE, Marchitelli LJ, Gordon SE, Harman EA, Mello R, et al. Effects of different heavy-resistance exercise protocols on plasma beta-endorphin concentrations. J Appl Physiol. 1993;74(1):450-9.
59. Kraemer WJ, Fry AC, Warren BJ, Stone MH, Fleck SJ, Kearney JT, et al. Acute hormonal responses in elite junior weightlifters. Int J Sports Med. 1992;13(2):103-9.

60. Walberg-Rankin J, Franke WD, Gwazdauskas FC. Response of beta-endorphin and estradiol to resistance exercise in females during energy balance and energy restriction. Int J Sports Med. 1992;13(7):542-7.

61. Pierce EF, Eastman NW, Tripathi HT, Olson KG, Dewey WL. Plasma beta-endorphin immunoreactivity: response to resistance exercise. J Sports Sci. 1993;11(6):499-52.

62. Kraemer RR, Acevedo EO, Dzewaltowski D, Kilgore JL, Kraemer GR, Castracane VD. Effects of low-volume resistive exercise on beta-endorphin and cortisol concentrations. Int $\mathrm{J}$ Sports Med. 1996;17(1):12-16.

63. Pierce EF, Eastman NW, McGowan RW, Tripathi H, Dewey WL, Olson KG. Resistance exercise decreases beta-endorphin immunoreactivity. Br J Sports Med. 1994;28(3):164-6.

64. Langenfeld ME, Hart LS, Kao PC. Plasma beta-endorphin responses to one-hour bicycling and running at $60 \%$ VO2max. Med Sci Sports Exerc. 1987;19(2):83-6.

65. Schwarz L, Kindermann W. Beta-endorphin, catecholamines, and cortisol during exhaustive endurance exercise. Int $\mathrm{J}$ Sports Med. 1989;10(5):324-8.

66. Panerai $A E$, Vecchiet $J$, Panzeri $P$, Meroni $P$, Scarone $S$, Pizzigallo $E$, et al. Peripheral blood mononuclear cell beta-endorphin concentration is decreased in chronic fatigue syndrome and fibromyalgia but not in depression: preliminary report. Clin J Pain. 2002;18(4):270-3.

67. Noakes T. Lore of running. Champaign, IL: Human Kinetics. 1991:408-25.

68. Schneider CD, Oliveira AR. Radicais livres de oxigênio e exercício: mecanismos de formação e adaptação ao treinamento físico. Rev Bras Med Esporte. 2004;10(4):308-13.

69. Schneider CD, Barp J, Ribeiro JL, Bello-Klein A, Oliveira AR. Oxidative stress after three different intensities of running. Can J Appl Physiol. 2005;30(6):723-34.

70. Oliveira AR, Schneider CD, Ribeiro JL, Deresz LF, Barp J, BelloKlein A. Oxidative stress after three different intensities of running. Med Sci Sports Exerc. 2003;35:S367.

Endereço para correspondência:

Giovani dos Santos Cunha

ESEF/UFRGS - Lapex

Rua Felizardo, 750

90690-200 Porto Alegre, RS

E-mail: giovanicunha@yahoo.com.br 